\title{
Influence of the Schwabe/Hale solar cycles on climate change during the Maunder Minimum
}

\author{
Hiroko Miyahara ${ }^{1}$, Yusuke Yokoyama ${ }^{2,3,4}$, and Yasuhiko T. \\ Yamaguchi ${ }^{2,3,4}$ \\ ${ }^{1}$ Institute for Cosmic Ray Research, The University of Tokyo, \\ 5-1-5 Kashiwanoha, Kashiwa 277-8582, Japan \\ email: hmiya@icrr.u-tokyo.ac.jp \\ ${ }^{2}$ Ocean Research Institute, The University of Tokyo, \\ 1-15-1 Minamidai, Nakano-ku, Tokyo 164-8639, Japan \\ ${ }^{3}$ Department of Earth \& Planetary Science, The University of Tokyo, \\ 7-3-1 Hongo, Tokyo 113-0033, Japan \\ ${ }^{4}$ Institute of Biogeoscience, Japan Agency for Marine-Earth Science and Technology, \\ 2-15, Natsushima-cho, Yokosuka 237-0061, Japan
}

\begin{abstract}
We have examined the variation of carbon-14 content in annual tree rings, and investigated the transitions of the characteristics of the Schwabe/Hale (11-year/22-year) solar and cosmic-ray cycles during the last 1200 years, focusing mainly on the Maunder and Spoerer minima and the early Medieval Maximum Period. It has been revealed that the mean length of the Schwabe/Hale cycles changes associated with the centennial-scale variation of solar activity level. The mean length of Schwabe cycle had been $\sim 14$ years during the Maunder Minimum, while it was $\sim 9$ years during the early Medieval Maximum Period. We have also found that climate proxy record shows cyclic variations similar to stretching/shortening Schwabe/Hale solar cycles in time, suggesting that both Schwabe and Hale solar cycles are playing important role in climate change. In this paper, we review the nature of Schwabe and Hale cycles of solar activity and cosmic-ray flux during the Maunder Minimum and their possible influence on climate change. We suggest that the Hale cycle of cosmic rays are amplified during the grand solar minima and thus the influence of cosmic rays on climate change is prominently recognizable during such periods.
\end{abstract}

Keywords. Sun: activity, solar-terrestrial relations, ISM: cosmic rays

\section{Introduction}

Both observational and proxy records of climate change often show quasi periodic variations similar to solar activity cycles over a wide range of time scales. However, the detailed mechanism and the extent of the influence of solar activity on climate change have not been clearly understood. Although the exact role of each of solar parameters on climate change has not been quantitatively clarified, several possible mechanisms are proposed; such as the forcing through total (e.g. Lean et al., 1995) and spectral irradiance (e.g. Haigh 1996; Kodera and Kuroda, 2005), solar wind (e.g. Tinsley, 1996) and the galactic cosmic rays (Friis-Christensen and Svensmark, 1997; Svensmark, 2007).

Among these parameters related to solar activity, galactic cosmic rays possess characteristic variations depending on the polarity of solar dipole magnetic filed as shown in Figure 1. The polarity of solar dipole magnetic field reverses at every maxima of 11-year sunspot activity cycle, and so the polarity reversals of solar magnetic field possess 22-year 


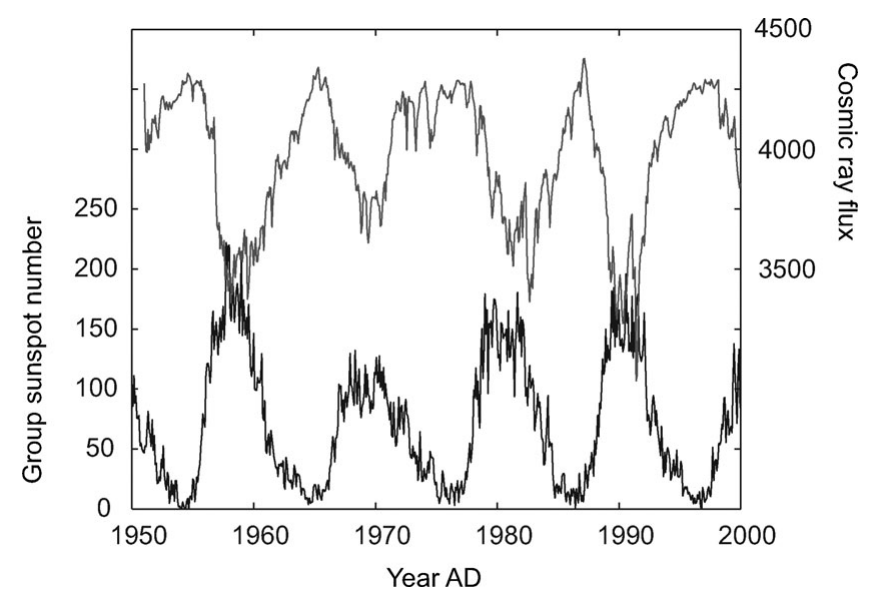

Figure 1. Monthly variation of the cosmic ray intensity observed by the Climax neutron monitor (upper gray line) and the group sunspot numbers (Hoyt \& Schatten, 1998) (lower black line).

cycle. The cosmic rays are modulated by solar wind and the interplanetary magnetic field and hence the flux of cosmic rays at the earth varies with the 11-year solar activity cycle, while, the polarity of solar dipole magnetic field determines the trajectory of cosmic rays in the heliosphere and thus the flux of cosmic rays at the earth varies depending also on the polarity of solar dipole magnetic field (Kota and Jokipii 2001). As is shown in Figure 1, the patterns of cosmic ray flux over solar cycles slightly differ depending on the polarity of solar dipole magnetic field, resulting in the component of 22-year cycle in cosmic-ray variation. This feature is very helpful in distinguishing the effect of cosmic rays on climate change from the other effects caused by e.g. irradiative outputs of the Sun.

Extension of the record of cosmic rays back in time enable us to examine if the connection between cosmic rays and climate change suggested by Friis-Christensen and Svensmark (1997) and Svensmark (2007) for the recent two decades had also existed in the past. We had investigated the history of Schwabe and Hale solar and cosmic ray cycles based on the carbon-14 content in tree rings with annual time resolution, originally for understanding the mechanism of multi-decadal to multi-centennial variation of solar activity level. Such record is however also applicable to investigating the Sun-climate relationship at decadal time scale. Carbon-14 is produced by cosmic rays, and circulates in the form of carbon dioxide to be absorbed in trees by photo synthesis. Since the age determination of each annual data is assured in the case of using tree rings, it is possible to determine the history of solar cycles with accurate timing. The beryllium-10 in ice cores from polar region can be also used for the reconstruction of solar cycles in the past. In the case of using ice cores, it is often difficult to obtain the record with absolute age, while, it is possible to derive much clear signal than carbon-14 due to the difference in the circulation process. The combination of these two nuclides provides clear image of cosmic ray variation with reliable age.

\section{Solar cycle during the Maunder Minimum}

Figure 2 shows the carbon-14 content in tree rings with decadal resolution obtained by Stuiver et al. (1998) and the annually measured data by Miyahara et al. (2004; 2006; 2007; 2008). During the last millennium, the Sun has experienced several prolonged sunspot minima such as the Maunder and the Spoerer Minima, which appear as upward peaks in 


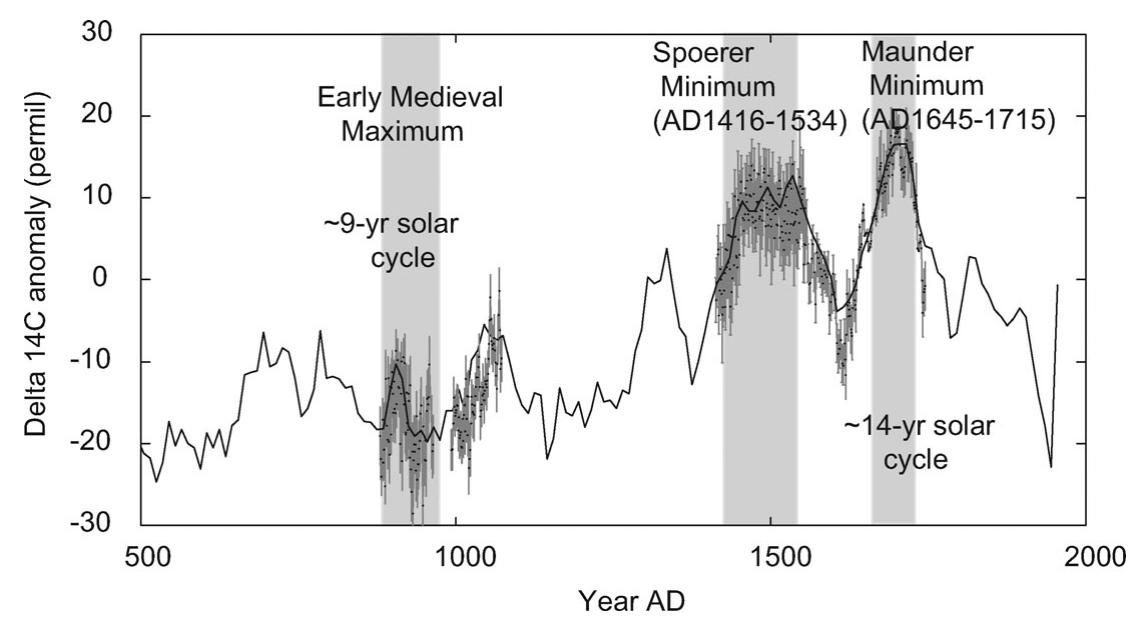

Figure 2. Variation of the carbon-14 content in tree rings with decadal resolution obtained by Stuiver et al., 1998 (black line), and the annually measured data by Miyahara et al. ( 2004; 2006, 2007; 2008) (black dots). The error bars for the annual data have been shown together (gray bars). The frequency analyses of the annual data over the shaded areas for the early medieval maximum and the Maunder Minimum have revealed that the mean length of the 11-year solar cycle had been modulated to $\sim 9$ years and $\sim 14$ years, respectively.

carbon-14 content in Figure 2, in addition to the long-term relatively active era called the Medieval Maximum Period (the $\sim 10-12$ th century). Frequency analyses of the annually measured carbon-14 records for the Maunder Minimum (AD1645-1715) revealed that the mean length of the 11-year cycle over the period had been stretched to be $\sin 14$ years (Miyahara et al., 2004; 2008). On the other hand, the mean length was sin9 years at the early Medieval Maximum Period (Miyahara et al., 2008). sin28-year cycle, which is the double length of $\sin 14$ years, was also detected in the frequency spectrum of carbon-14 content during the Maunder Minimum, suggesting that the polarity reversals of solar dipole magnetic field had been carried on during the prolonged sunspot absence. Figure 3 shows the reconstructed Schwabe and the Hale cycles during the Maunder Minimum. The Schwabe cycle in Figure 3(a) was obtained by applying Fourier band-pass filter with the bandwidth of 10-16 years, while the Hale cycle in Figure 3(b) was obtained by applying the filter of 20-30 years. The reconstructed Schwabe cycle is consistent with the variation of group sunspot numbers by Hoyt and Schatten (1998), at the times they show distinct cyclic variation. Solar cycles can now be extended back to the early 17 th century complemented by the carbon-14 record, revealing that the Maunder Minimum had started at Solar Cycle -8.

\section{Solar and cosmic-ray Hale cycle during the Maunder Minimum}

The Hale cycle of cosmic rays in the recent decades indicate that the mean flux of cosmic rays over solar cycle is relatively higher when the polarity of solar dipole magnetic filed is positive as shown in Figure 1. This profile is consistently reproduced by the numerically simulated cosmic ray flux as shown in Figure 4(b), where the profile is obtained based on the model by Kota and Jokipii (2001) shown in Figure 4(a) by assuming that the range of the tilt angle of solar neutral line over solar cycle is 10-70 degrees. For the Maunder Minimum, it had been suggested by Jokipii (1991) that the phase of the Hale cycle could have been 180 degrees reversed during the Maunder Minimum, which means that incoming cosmic ray flux is expected to be relatively higher when the polarity is 
(a)

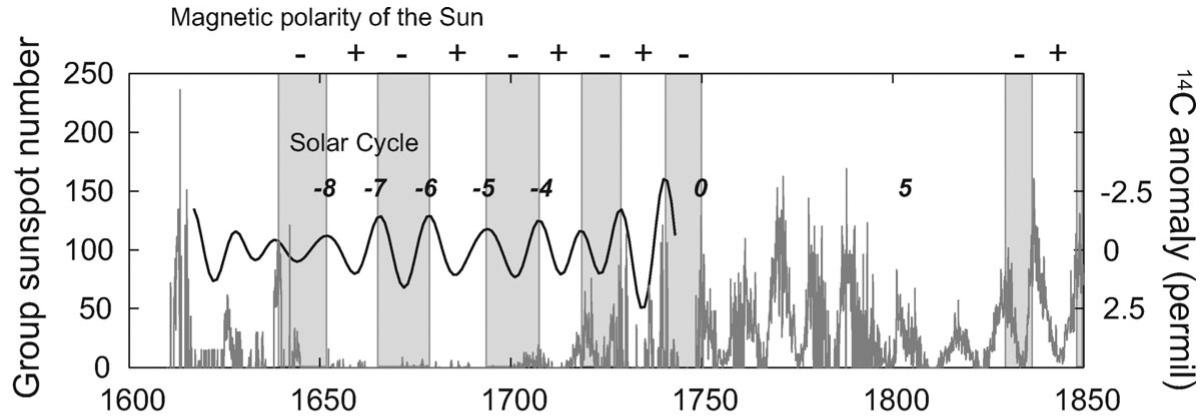

(b)

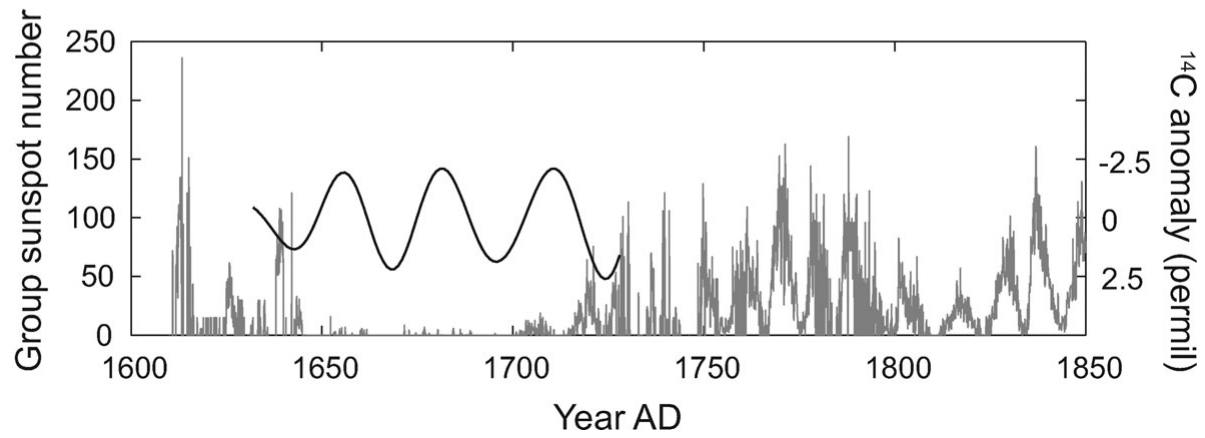

Figure 3. Reconstructed Schwabe and Hale cycles based on annually measured carbon-14 content in tree rings by Miyahara et al. $(2004 ; 2007)$. (a) Band-pass filtered carbon-14 record with the bandwidth of 10-16 years, together with the group sunspot numbers by Hoyt \& Schatten (1998) (gray line). The number of Solar Cycle is determined based on the band-pass filtered carbon-14 record. (b) Same with (a) but the bandwidth is 20-30 years. The magnetic polarity shown in (a) is determined by the phase of Hale cycle in (b) for the Maunder Minimum, while that for AD1830-1850 is determined by the number of solar cycles from present.

negative. Based on the variations of the Schwabe/Hale cycles detected by carbon-14 and also on the model in Figure 4(a), we obtained the time profile shown in Figure 4 (c) as the most probable variation of cosmic rays during the Maunder Minimum, where the range of the tilt angle was assumed to be 0-70 degrees. The profile is characterized by the sharp peak of cosmic ray flux at solar cycle minima when the magnetic polarity of the Sun is negative, resulting in the enhancement of the Hale cycle in cosmic ray variation. This profile has been recently supported by the beryllium-10 record from Greenland ice core (Berggren et al., 2009). In the record of beryllium-10, three sharp peaks are recognized at the beginning of the Maunder Minimum, 1700AD and in between. The periods of these three peaks are consistent with the timing that the content of carbon-14 in tree rings was also found to be relatively higher as is shown in Figure 3(a). It suggests that these three periods correspond to the minima of Schwabe cycle of the times solar magnetic polarity is negative. Both of our carbon-14 and the Greenland beryllium-10 records suggest for the Maunder Minimum that the tilt angle of solar neutral line had reached close to $\sim 0$ degree at every cycle minima, while the neutral line had certainly waved toward the suggested polarity reversals at solar cycle maxima. The transitions of the polarity of solar dipole magnetic field around the Maunder Minimum deduced from the carbon-14 record is summarized in Figure 3(a). The determined polarity is consistent with what we obtain from the counting of the number of solar cycles from present (as those for AD1830-1850 in Figure 3(a)). It had been pointed out that there is a possibility that Solar Cycle 4 
(a)
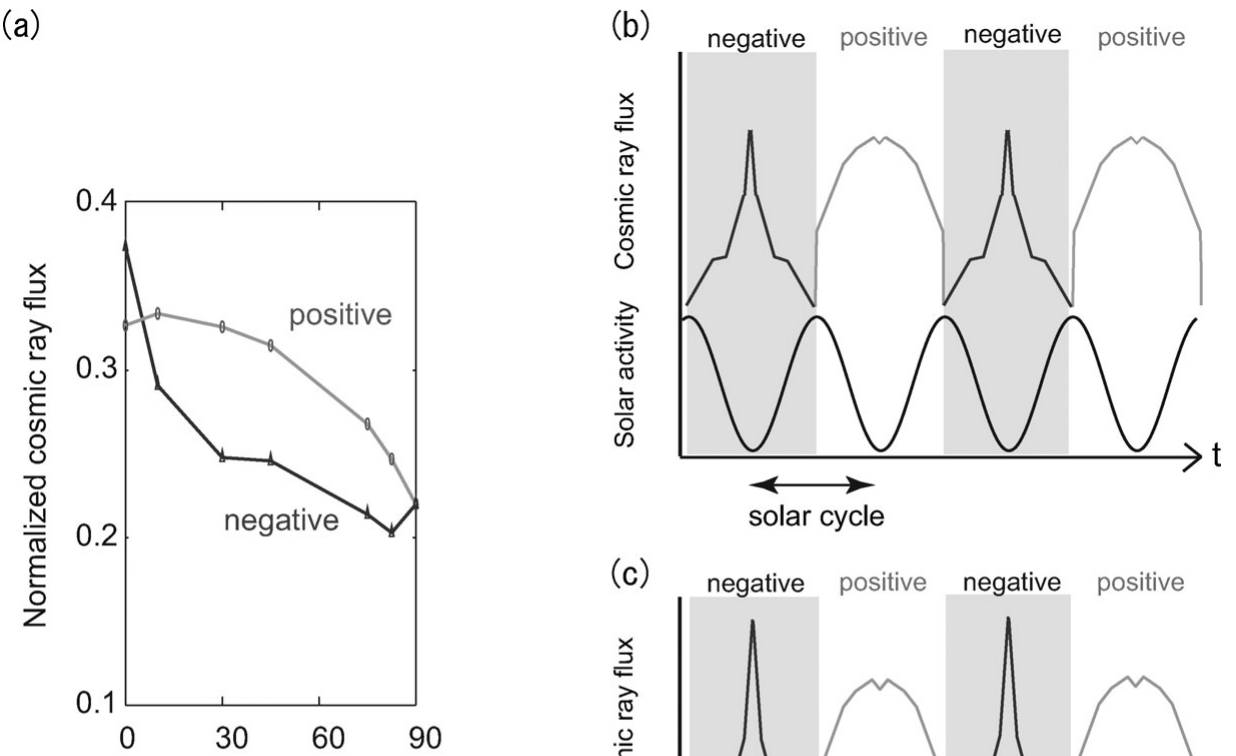

Tilt angle (degrees)

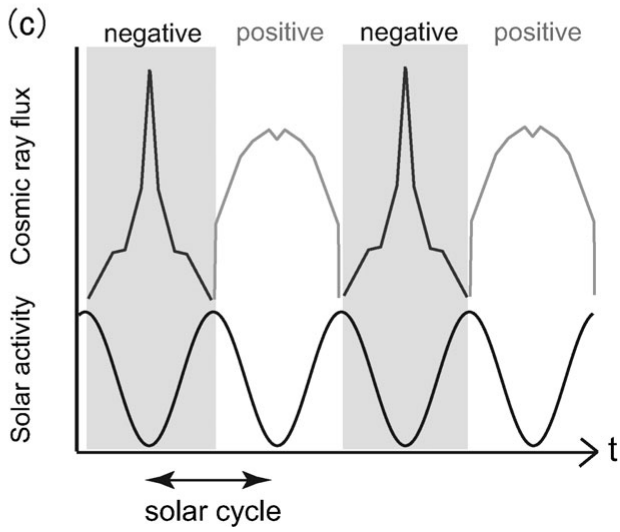

Figure 4. Numerically simulated variation of cosmic ray flux at the earth against solar activity and magnetic polarity by Kota and Jokipii (2001). (a) The flux of cosmic rays against the polarity of solar dipole magnetic field and the tilt angle of solar magnetic neutral line (based on Kota \& Jokipii, 2001). (b) Model of the cosmic ray variation when the range of the tilt angle over solar cycle is 10-70 degrees, which is comparable to that of recent decades. (c) Model of the cosmic ray variation during the Maunder Minimum where the range of the tilt angle is assumed to be 0-70 degrees.

consists of two solar cycles (Usoskin et al., 2001), however, the determined history of the polarity suggests that it consists of single solar cycle with long duration.

\section{Influence of the Schwabe/Hale solar cycles on climate change}

Figure 5 shows the comparison between the reconstructed solar cycle (same as in Figure $3(\mathrm{a})$ ) and the reconstructed temperature based on oxygen-18/oxygen-16 ratio in Greenland Ice core (Vinther, 2003). The shaded areas correspond to cycle minima of negative solar polarity determined by carbon-14 record, where the flux of incoming galactic cosmic rays is suggested to be high as discussed in the previous section. It is recognized that the reconstructed temperature in Figure 5(b) shows rapid cooling around the shaded area, corresponding to the periods when the flux of cosmic rays is high. It is suggested that the climate change has some component dependent on the polarity of solar magnetic field, resulting in the appearance of the Hale cycle in its variation, and that solar magnetic cycle is playing important role in climate change probably through the mechanism involving cosmic rays. As the actual length of the Schwabe cycle is $\sim 14$ years, 


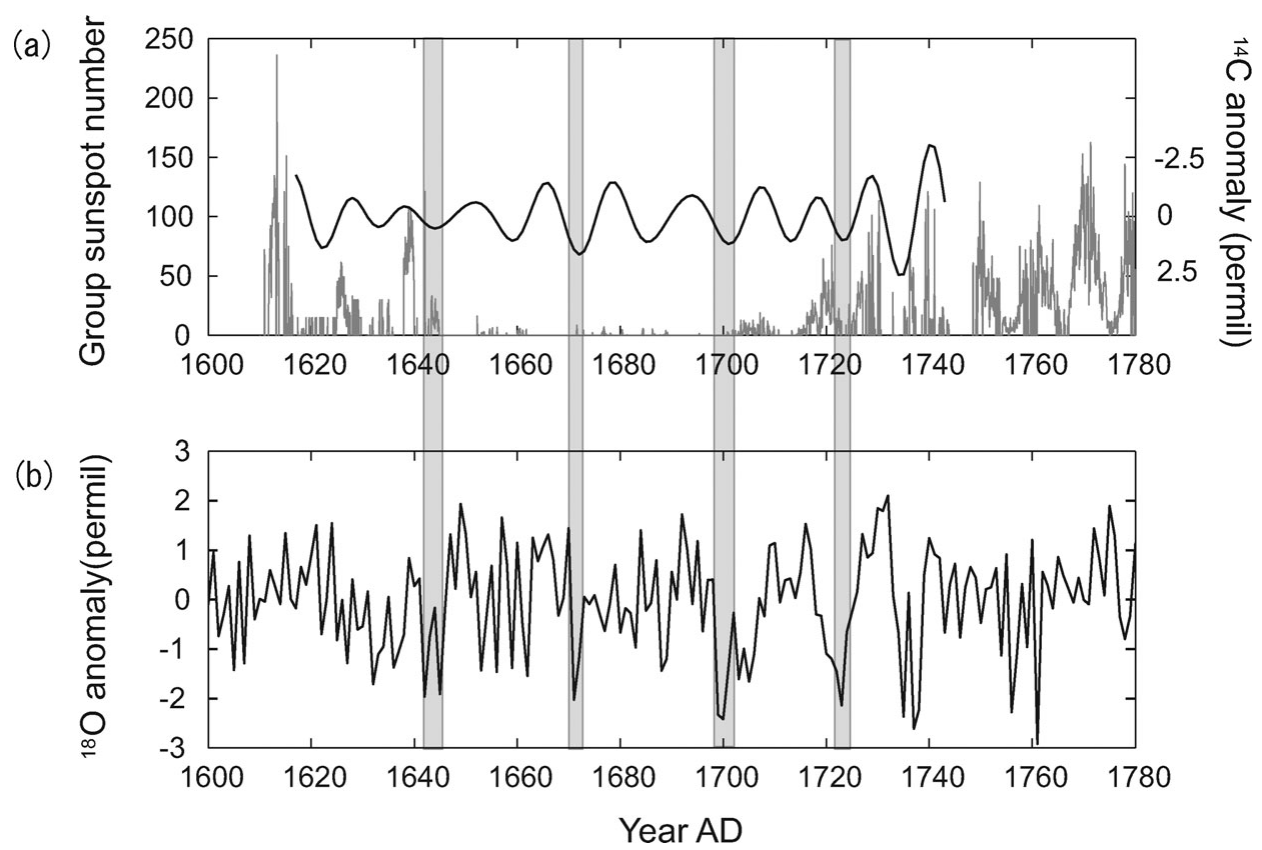

Figure 5. Comparison of solar cycle with reconstructed temperatures around the Maudner Minimum. (a) Reconstructed Schwabe cycle based on the band-pass filtered carbon-14 record as shown in Figure 3(a), together with the group sunspot numbers. (b) Reconstructed temperature based on oxygen-18/oxygen-16 ratio in the Greenland ice core by Vinther et al. (2003). The shaded areas correspond to the solar cycle minima of polarity negative determined by carbon-14 record as shown in Figure 3.

the length of the Hale cycle detected in the reconstructed temperature is also stretched to be $\sim 28$ years. As has been discussed in our previous paper (Miyahara et al., 2008), the phase of the Hale cycle in climate change is reversed after the Maunder Minimum as well as that of cosmic rays, and the cooling is found to occur especially around the cycle minima of polarity positive. The length of climate Hale cycle also recovers to 22 years. It is noteworthy that the variability of climate change caused by cosmic ray variation can be more prominent during the grand solar minima compared to the other era (also see Miyahara et al., 2008), since the variability of cosmic rays is likely enhanced during such periods, as is suggested in Figure 4(c), due to the change in the range of tile angle of solar neutral line. It is contradicting to the expected feature of the influence of irradiative outputs of the Sun, for which it is expected that the amplitude becomes less when solar activity is low. Such amplification of the "22-year" cycle could have brought additional occasional very severe climate during the Little Ice Age.

\section{Summary}

The mechanism of the influence of cosmic rays on the cloud formation is not fully understood, however, our proxy based analyses of cosmic rays and climate change during the Maunder Minimum exhibit the importance of cosmic rays as a medium of solar forcing of climate change at decadal to multi-decadal time scales. The complex features of solar magnetic and cosmic ray cycles, such as the variable length of the "11-year" cycle, the subsequent lengthening/shortening of the "22-year" Hale cycle, the amplification of 
the 22-year cycle in cosmic rays at grand solar minima, may be able to explain some of the complex features of climate change at this time scale.

\section{Acknowledgements}

We thank Dr. J. Kota (Univ. of Arizona) and Prof. K. Munakata (Shinshu Univ.) for fruitful discussions. This research was partly funded by the Japan Society for the Promotion of Science, the Grant-in-Aid for Scientific Research, and Global Environmental Research Fund.

\section{References}

Berggren, A.-M., Beer, J., Possnert, G., Aldahan, A., Kubik, P., Christl, M., Johnsen, S. J., Abreu, J., \& Vinther, B. M. 2009 Geophys. Res. Lett., 36, L11801

Friis-Christensen, E. F. \& Svensmark, H. 1997 Adv. Space Res., 20, 913

High, J. D. 1996 Science, 272, 981

Hoyt, D. V. \& Schatten, K. H. 1998 Sol. Phys., 181, 491

Jokipii, J. R. 1991, in: C.P. Sonnet, M.S. Giampapa \& M.S. Matthers (eds.), The Sun in Time, (The University of Arizona Press, USA)

Kodera, K. \& Kuroda, Y. 2005 J. Geophys. Res., 110, D02111

Kota, J. \& Jokipii, J. R. 2001 Adv. Space Res., 27, 529

Lean, J. L., Beer, B., \& Bradley, R. 1995 Geophys. Res. Lett., 22, 3195

Miyahara, H., Masuda, K., Muraki, Y., Furuzawa, H., Menjo, H., \& Nakamura, T. 2004 Sol. Phys., 224, 317

Miyahara, H., Masuda, K., Muraki, Y., Kitagawa, H., \& Nakamura, T. 2006 J. Geophys. Res., 111, A03103

Miyahara, H., Masuda, K., Nagaya, K., Kuwana, K., Muraki, Y., \& Nakamura, T. 2007 Adv. Space Res., 40, 2060

Miyahara, H., Yokoyama, Y., \& Masuda, K. 2008 Earth Planet. Sci. Lett., 272, 290

Stuiver, M., Reimer, P., \& Braziunas, T.F. 1998 Radiocarbon, 40, 1127

Svensmark, H. 2007 Astron. Geophys., 48, 118

Tinsley, B. A. 1996 J. Geophys. Res., 101, 29701

Usoskin, I. G., Mursula, K., \& Kovaltsov, G.A. 2001 A\&A, 101, 29701

Vinther, B. M., Johnsen, S. J., Andersen, K. K., Clausen, H. B., \& Hansen, A. W. 2003 Geophys. Res. Lett., 30, doi:10.1029/2002GL016193 